\title{
Right and left-sided colon cancers - specificity of molecular mechanisms in tumorigenesis and progression
}

\author{
Kavitha Mukund', Natalia Syulyukina', Sonia Ramamoorthy ${ }^{2}$ and Shankar Subramaniam³,3*
}

\begin{abstract}
Background: Given the differences in embryonic origin, vascular and nervous supplies, microbiotic burden, and main physiological functions of left and right colons, tumor location is increasingly suggested to dictate tumor behavior affecting pathology, progression and prognosis. Right-sided colon cancers arise in the cecum, ascending colon, hepatic flexure and/or transverse colon, while left-sided colon cancers arise in the splenic flexure, descending, and/or sigmoid colon. In contrast to prior reports, we attempt to delineate programs of tumorigenesis independently for each side.

Methods: Four hundred and eleven samples were extracted from The Cancer Genome Atlas-COAD cohort, based on a conservative sample inclusion criterion. Each side was independently analyzed with respect to their respective normal tissue, at the level of transcription, post-transcription, miRNA control and methylation in both a stage specific and stage-agnostic manner.

Results: Our results indicate a suppression of enzymes involved in various stages of carcinogen breakdown including CYP2C8, CYP4F12, GSTA1, and UGT1A within right colon tumors. This implies its reduced capacity to detoxify carcinogens, contributing to a genotoxic tumor environment, and subsequently a more aggressive phenotype. Additionally, we highlight a crucial nexus between calcium homeostasis (sensing, mobilization and absorption) and immune/GPCR signaling within left-sided tumors, possibly contributing to its reduced proliferative and metastatic potential. Interestingly, two genes SLC6A4 and HOXB13 show opposing regulatory trends within right and left tumors. Post-transcriptional regulation mediated by both RNA-binding proteins (e.g. NKRF (in left) and MSI2 (in right)) and miRNAs (e.g. miR-29a (in left); miR-155, miR181-d, miR-576 and miR23a (in right)) appear to exhibit side-specificity in control of their target transcripts and is pronounced in right colon tumors. Additionally, methylation results depict location-specific differences, with increased hypomethylation in open seas within left tumors, and increased hypermethylation of $\mathrm{CpG}$ islands within right tumors.
\end{abstract}

Conclusions: Differences in molecular mechanisms captured here highlight distinctions in tumorigenesis and progression between left and right colon tumors, which will serve as the basis for future studies, influencing the efficacies of existing and future diagnostic, prognostic and therapeutic interventions.

Keywords: Left-sided colon cancer, Right-sided colon cancer, Biomarkers, Methylation, miRNA, RNA binding proteins, Xenobiotic metabolism, GPCR signaling, Non-synchronous tumors

\footnotetext{
* Correspondence: shankar@ucsd.edu

'Department of Bioengineering, University of California, San Diego, La Jolla, CA, USA

${ }^{3}$ Department of Cellular and Molecular Medicine, University of California, San Diego, La Jolla, CA, USA

Full list of author information is available at the end of the article
}

(c) The Author(s). 2020 Open Access This article is licensed under a Creative Commons Attribution 4.0 International License, which permits use, sharing, adaptation, distribution and reproduction in any medium or format, as long as you give appropriate credit to the original author(s) and the source, provide a link to the Creative Commons licence, and indicate if changes were made. The images or other third party material in this article are included in the article's Creative Commons licence, unless indicated otherwise in a credit line to the material. If material is not included in the article's Creative Commons licence and your intended use is not permitted by statutory regulation or exceeds the permitted use, you will need to obtain permission directly from the copyright holder. To view a copy of this licence, visit http://creativecommons.org/licenses/by/4.0/ The Creative Commons Public Domain Dedication waiver (http://creativecommons.org/publicdomain/zero/1.0/) applies to the data made available in this article, unless otherwise stated in a credit line to the data. 


\section{Background}

Colorectal cancer $(\mathrm{CRC})$ is a heterogeneous disease with distinct clinical, molecular, and pathophysiological characteristics. The Surveillance, Epidemiology and End Result (SEER) program of the National Cancer Institute, in 2018, identified CRC as the fourth most prevalent cancer, and the third leading cause of mortality due to cancers, with an average five-year survival of $64.5 \%$. Traditionally, patient subgroups in CRC are associated with dysfunction of mismatch repair genes (microsatellite instable group, MSI); KRAS, APC and/or BRAF mutations (chromosomal instability group) and hypermethylation (CpG island methylation phenotype, CIMP) [1]. However, increasing evidence on the heterogeneity of these genetic and epigenetic changes, necessitated a model for identifying consensus molecular subtypes (established in 2015) [2]. It is now widely acknowledged that the heterogeneity extends beyond the above recognized molecular mechanisms. Location of tumor within the colon is gaining traction as crucial factor in determining disease progression, prognosis and management, and begs the question if colon and rectal cancers can be treated as being mechanistically similar [3]. To this extent we focus our attention on discerning the molecular mechanisms governing colon cancer, particularly tumors arising in the left and right colons.

Right-sided colon cancers (RSCC or proximal tumors) occur in the cecum, ascending colon, hepatic flexure and/ or transverse colon, while left-sided colon cancers (LSCC or distal tumors) arise in the splenic flexure, descending colon, and/or sigmoid colon. This distinction has been observed at physiological, molecular and therapeutic levels for RSCC and LSCC [4]. For example, from the perspective of disease management, National Comprehensive Cancer Network (NCCN) Clinical Practice Guidelines in Oncology outline differences in therapeutic sensitivities of targeted drugs differ between sites in CRC for e.g. EGFR inhibitors, such as cetuximab, panitumumab, have been indicated to be less effective as first-line therapy for RSCC metastatic disease [5]. At a physiological level, RSCC patients are more likely to exhibit advanced tumor stage, increased tumor sizes, frequently poorly differentiated tumors, with increased lymphovascular invasion than LSCC patients [6]. Systematic reviews and meta-analyses have correlated RSCC with poor prognosis and overall survival [7]. At a molecular level, multiomics analyses have identified differences between RSCC and LSCC, including differences in miRNA control and transcriptional regulation and its immune landscape [8]. RSCC also tend to exhibit different mutational burdens and increased hypermethylation compared to LSCC [9].

To the best of our knowledge, recent studies focusing on molecular differences between RSCC and LSCC, have done so by directly comparing the two etiologies (e.g. [10]). However, tumor transforms the state of healthy tissue, and we would benefit by comparing tumor tissue with its normal first, and then extend the understanding to side specific differences in the etiology of colon cancer. The analyses outlined in this paper is grounded in this simple idea and focuses on mechanistic differences leading to the pathogenesis of these two cancer types. Towards this, we perform an integrative analysis using publicly available colon cancer (COAD) data (from nonsynchronous tumor patients) in The Cancer Genome Atlas (TCGA) project. We first establish transcriptional regulation in both RSCC and LSCC, independently, compared to their respective normal tissue and identify common and distinct programs of regulation that exist between proximal and distal tumorigenesis. We next systematically identify and characterize roles for miRNAs and RNA binding proteins, in colon cancer, in a side specific manner. Differences in epigenetic (DNA methylation) profiles, and its impact on the observed gene regulation, within both of these colon cancer types are also comprehensively analyzed.

\section{Methods}

\section{Sample inclusion criteria}

TCGA level 3 COAD raw count data for 499 solid tumor and normal tissue was downloaded via Firebrowse in July 2018. Samples were classified into right/proximal and left/distal based on their site of extraction. Since TCGA clinical data was not specifically annotated for exact location along the transverse colon, all samples annotated "transverse colon" were excluded from analysis [11]. Samples with discrepancy in site of extraction, and with a history of "synchronous" colon tumors and/or adjuvant therapy were also excluded. 411 samples (170 LSCC and 17 Left normal and 203 RSCC and 21 Right normal) were subsequently utilized for RNAseq analysis. Baseline characteristics of the sample patient cohort is outlined in Supplementary Table S1.

\section{RNAseq analyses}

Count matrix normalization and differential analysis was performed using the "DEseq2" package available through R/Bioconductor [12]. Expression of 17,597 genes was used as input after basic prefiltering. Differentially expressed genes (DEGs) for each side were identified at $|\log 2|$ fold change $\left(\mathrm{fc}_{\mathrm{c}}\right)>1$ and adj p-val $<0.05$ (Benjamini-Hochberg). We reasoned that the thresholds chosen here were optimal to identify biologically relevant targets for the purposes of this analysis (Supplementary Table S2). Stage specific analysis (utilizing AJCC staging information extracted from the TCGACOAD clinical metadata) was also likewise performed (Supplementary Tables S3 and S4). High confidence (> $0.9)$ protein-protein interactions (PPI) was downloaded from STRING database (v10.5). Side specific PPIs were 
extracted corresponding to DEGs on each side. Coexpression between genes was utilized as weights to cluster side-specific networks using MCL clustering. The mSVM-RFE method with 10 fold cross-validation [13] was utilized to identify DEGs, that improved classification efficiency of side-specific tumors from normal tissue (Supplementary Table S5).

\section{Cox regression analyses}

Survival outcome modeled results with reference to patient overall survival (OS). Specifically, events were defined as death by any cause, and time was accurate to the day. $p$-values were obtained from univariate Cox proportional-hazards regression models for the entire list of differentially expressed genes on the right and left (using the $\mathrm{R}$ packages Regparallel and Survival). All Kaplan-Meier survival curves were constructed using survival and ggplots2 packages. Median expression was utilized to separate high from low expression group in left and right samples. A total of 76 and 42 gene markers were identified to be associated with overall survival in left and right respectively (logrank p-val $<0.01$; Supplementary Table S6).

\section{miRNA analyses}

miRNA expression data was downloaded using TCGAbiolinks [14], across 449 annotated samples (see Methods), of which a total of 370 patient samples met the inclusion criteria. Differentially expressed miRNAs (DEMs) for all pairwise comparisons were identified using DESeq2 (adj $p<0.05$ ). Manually curated miRNAtarget interactions from miRTarBase 7.0 [15] between DEGs and DEMs were extracted. Subsequently the left network contained 250 connected nodes, with 211 interactions (Supplementary Figure 1) and the right contained 319 connected nodes with 225 interactions (Supplementary Figure 2). Spearman rank correlation between miRNA and mRNA expression in tumor samples was added as edge weights. The networks were clustered on these weights (using MCL clustering) to ascertain clusters relevant to RSCC and LSCC.

\section{Quantification of differential alternative splicing}

Percent splice-in (PSI) values were downloaded from TCGASpliceSeq (with PSI values in at least 75 samples) for our sample cohort, accounting for a total of 23,176 events. "psichomics" [16] available through Bioconductor/R was utilized to extract significantly differentially alternatively spliced (AS) events between tumor and normal samples for each side independently. Seven different event types were detected between normal and tumor samples for each side namely, Exon Skip (ES), Alternate Donor Sites (AD), Alternate Acceptor Sites (AAs), Retained Intron (RI), Alternate Promoter (AP),
Alternate Terminator (AT) and Mutually Exclusive Exons (ME) [17]. Significantly differentially AS events were defined as events with a $\mid \Delta$ median PSI $\mid \geq 0.1$ and FDR $\leq 0.01$ (Supplementary table S10) [16]. Of all the events detected, only events occurring in DEGs were considered significant for our current analysis and chosen for further study (henceforth referred to as "sigAS" events).

\section{Identifying key RNA-binding proteins}

A combined list of 1608 RNA-binding proteins (RBPs) were extracted from supplementary tables available in two prior publications [18, 19], consolidating known RBPs. We referenced this RBP list against the list of DEGs identified in LSCC and RSCC to identify differentially regulated RBPs $(\log 2 \mathrm{fc}>1$, adj $p<0.05)$ for each side independently. We utilized the entire list of DEGs on each side to identify enrichment of RBP binding using AURA2 [20]. Since, we were particularly interested in RBPs that contribute to sigAS, we extracted 295 splicing associated RBPs (with GO RNA related category description containing keyword "splicing" from [19]). 4 and 5 RBPs were identified to be differentially regulated in RSCC and LSCC, respectively, and were considered for further analysis. Spearman correlation between expression (transcripts per million, downloaded from Firebrowse as before) of the differentially expressed splicing associated RBPs against PSI values for sigAS events was performed within tumor samples, on left and right. Clustering was performed using MCL clustering.

\section{Methylation analyses}

Illumina HumanMethylation450 beadchip data for TCGA-COAD, probing nearly $480 \mathrm{k}$ CpG sites, was downloaded using TCGAbiolinks. Nearly $370 \mathrm{k}$ probes were retained for each side, after eliminating probes on sex and "NA" chromosomes. Mean methylation and differentially methylated probes (DMPs) were both determined using the TCGAbiolinks package, independently for each side. DMPs were identified using $\beta$ (average promoter methylation value \%) that were at least $25 \%$ differential $(|\Delta \beta|>0.25)$ between tumor and adjacent normal tissues with adj $p \leq 0.001$, for each side. Correlated and anti-correlated pairs of DEGs with DMPs (Hypo and Hyper) and transcription factor (TF) enrichment were identified using ELMER [21].

\section{Enrichment and visualization}

All functional enrichment analyses were performed using mSigDB's hallmark data sets and GO ontologies. All networks were constructed, clustered, annotated and analyzed in Cytoscape [22]; Visualization was performed using clusterProfiler [23]. Transcription factor enrichment of targets was performed using $\mathrm{ARCH}^{4}$ database [24] or ELMER where applicable. 


\section{Results}

Establishing transcriptional regulation in RSCC and LSCC Differential analysis identified 2495 DEGs in LSCC, with respect to left normal colon tissue, and 2589 DEGs in RSCC, with respect to right normal colon tissue (Fig. 1a). 957 (up) and 975 (down) DEGs are identified to be commonly regulated in both RSCC and LSCC while, 655 and 561 DEGs are "uniquely" regulated in RSCC and LSCC, respectively (Fig. $1 \mathrm{~b}$ and $\mathrm{c}$ ).

\section{A common program of tumorigenesis exists between right and left sided colon tumors}

Malignant tumor cells are highly plastic and are characterized by alterations in metabolism, adhesion, proliferation and migration, requiring coordinated activity of several signaling pathways and mechanisms. Our results indicate that the large overlap of "commonly" regulated DEGs that exist between RSCC and LSCC fit broadly into these categories and are frequently seen dysregulated in colon cancers (Fig. 2a). For instance, dysregulation of $\mathrm{WNT} / \beta$ catenin pathway genes affecting proliferative potential of CRC is evident in both, with the upregulation of several WNT pathway genes including AXIN2, WNT2, WNT3, WNT7B, DKK1/4, NKD1/4, TCF7, MYC and NOTUM. NOTUM, a glypican-dependent WNT inhibitor serves as a negative feedback regulator for WNT activation [25], and is associated with the progression of CRC [26]. We identified NOTUM to be significantly associated with OS in patients with both RSCC (Hazard Ratio 95\% CI - 0.44 (0.24-0.82), logrank $p<0.01$ ) and LSCC (HR 95\% CI 3.23 (1.27-8.2), $\mathrm{p}<0.01)$ indicating that a higher expression favors LSCC while lower expression favors RSCC (Fig. 2b). Other frequently dysregulated genes including $A P C, G S K 3 B$ were identified as commonly dysregulated (albeit below our fc threshold).

Cellular metabolism is tightly linked with cellular growth and proliferation of tumors. Loss of AMPactivated protein kinase ( $A M P K 1)$ activity can drive reprogramming of cellular metabolism and is at the center of the network regulating cell growth and proliferation (via TP53). AMPK1 driven-metabolic dis-homeostasis becomes evident by the observed dysregulation of melanoma antigens (MAGEA2/A3/A6) [27], within both these tumor types. The acidic and hypoxic tumor microenvironment also influences survival and proliferative potential. Carbonic anhydrases (CA), metalloenzymes which catalyze reversible hydration of $\mathrm{CO}_{2}$, have been identified as crucial mediators of tumor $\mathrm{pH}$ [28]. Notably, several cytosolic CAs (such as CA1/2/4) and water channels $(A Q P 8)$ are suppressed in both, alluding to the

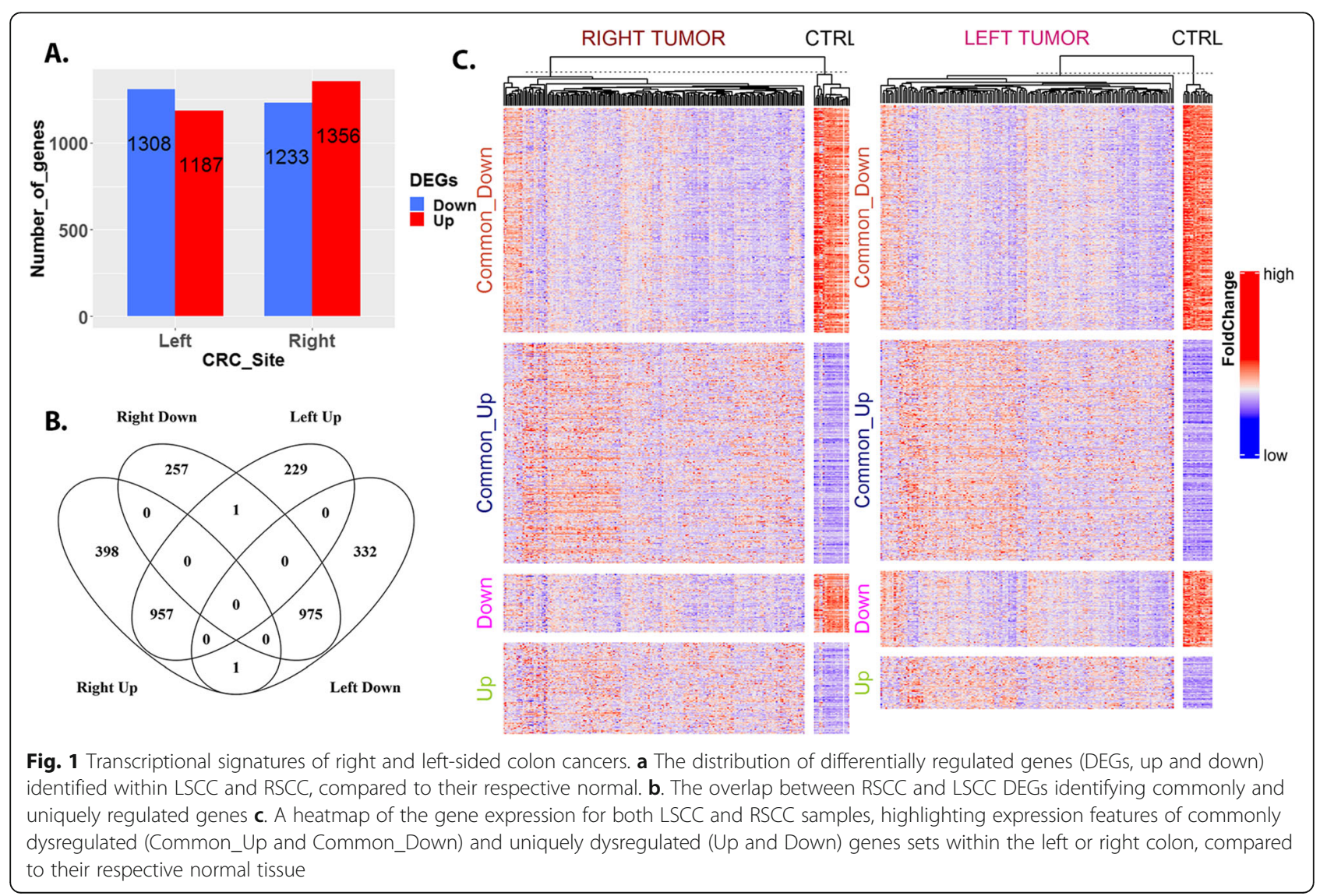




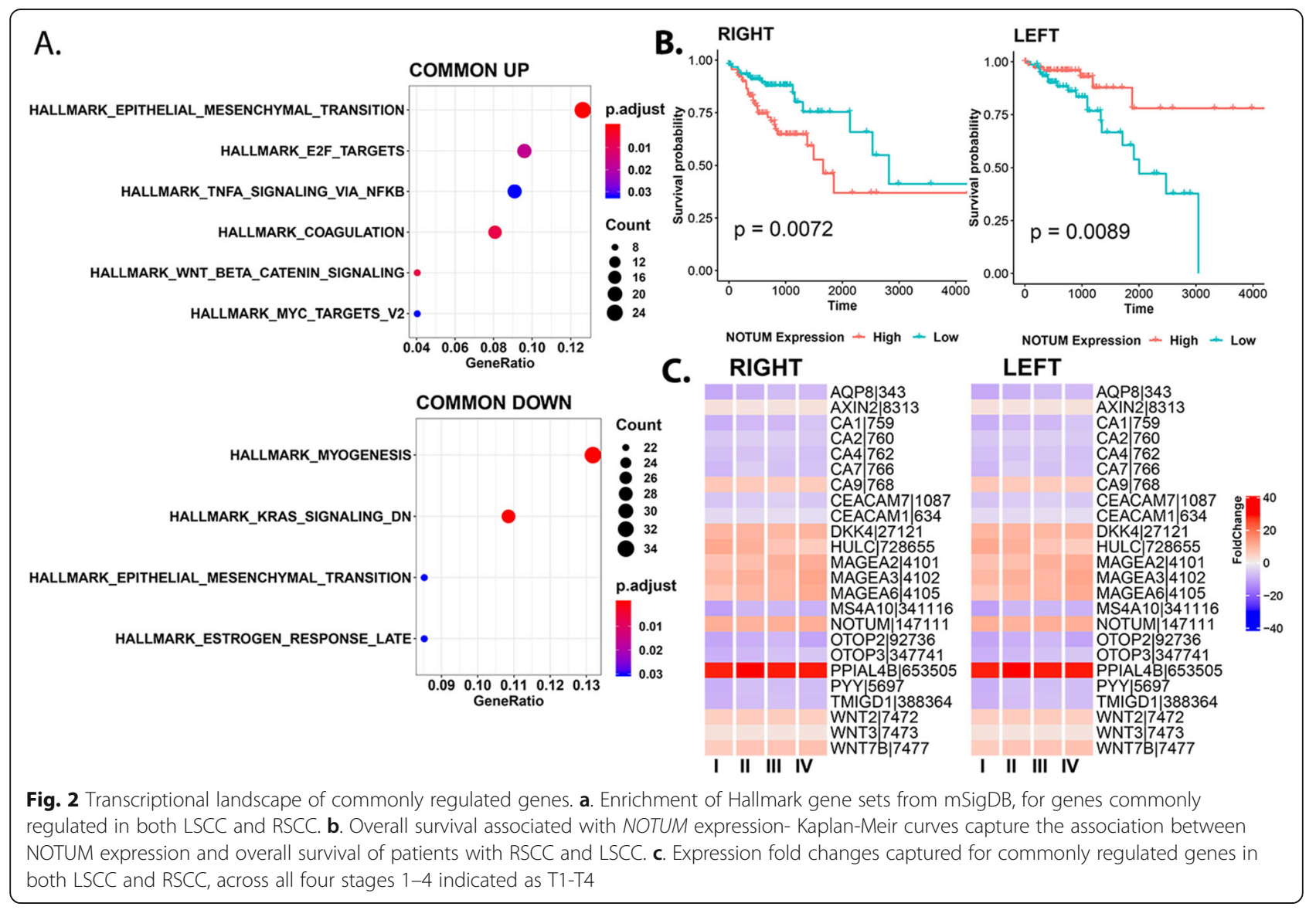

reduced availability of the universal buffer $\mathrm{HCO}_{3}{ }^{-}$. It has been previously postulated that extracellular CAs such as $C A 9$ (upregulated in both tumor types), act to raise the extracellular $\mathrm{pH}$ favoring tumor cell growth, proliferation, and survival [29]. Several other markers including FDA approved CRC biomarkers such as CA125 (MUC16) and CEA (carcino-embyonic antigen, CEACAM1/7) are upregulated in both RSCC and LSCC.

Interestingly, several genes whose precise molecular interactions are yet to be completely understood, are among the most highly expressed genes in both proximal and distal tumors including OTOP2 (controlled by wild-type TP53) [30], OTOP3, PYY and PPIAL4; and many with limited supporting evidence, might serve as interesting candidates for future research in colon cancers (Supplementary Table S7). Additionally, several genes discussed herein are regulated across all stages further highlighting their role in the evolution and maintenance of tumors over time, in a side-independent manner (Fig. 2c).

\section{Right-sided colon tumors exhibit altered lipid, bile and xenobiotic metabolism}

Liver is largely considered the major organ for biotransformation (chemical detoxification and metabolism).
However, there is increasing acknowledgement of extrahepatic biotransformation (especially in the gastrointestinal (GI) tract) and its association with GI carcinogenesis. Several families of enzymes are associated with various stages of breakdown of carcinogens within the human body including cytochrome P450 (CYP), glutathione Stransferase (GSTA1), and UDP-glucuronosyltransferase (UGT) superfamily [31]. Notably, we identify these gene families to be enriched among gene-sets suppressed in RSCC (Fig. 3a). Particularly, we identify a suppression of enzymes from $\mathrm{CYP} 2 \mathrm{C}$ and $4 \mathrm{~F}$ families (CYP2C8, CYP2C18 and CYP4F12, Fig. 3b). These results are interesting in light of a recent study [32], which identified contrasting results with an upregulation of CYP2C family of enzymes in animal models of CRC. The CYP2C pathway enzymes convert arachidonic acid (AA) into active epoxyeicosatrienoic acids (EETs), while CYP4F family convert AA to hydroxyl EETs, both compounds suggested to promote carcinogenesis in certain contexts. UGT proteins catalyze the glucuronidation reaction, allowing for the utilization and/or detoxification of necessary chemicals [33]. We identify suppression of several UGT1A isoforms in tumor compared to normal, particularly the extrahepatic isoforms UGT1A10/A7 and A8. 


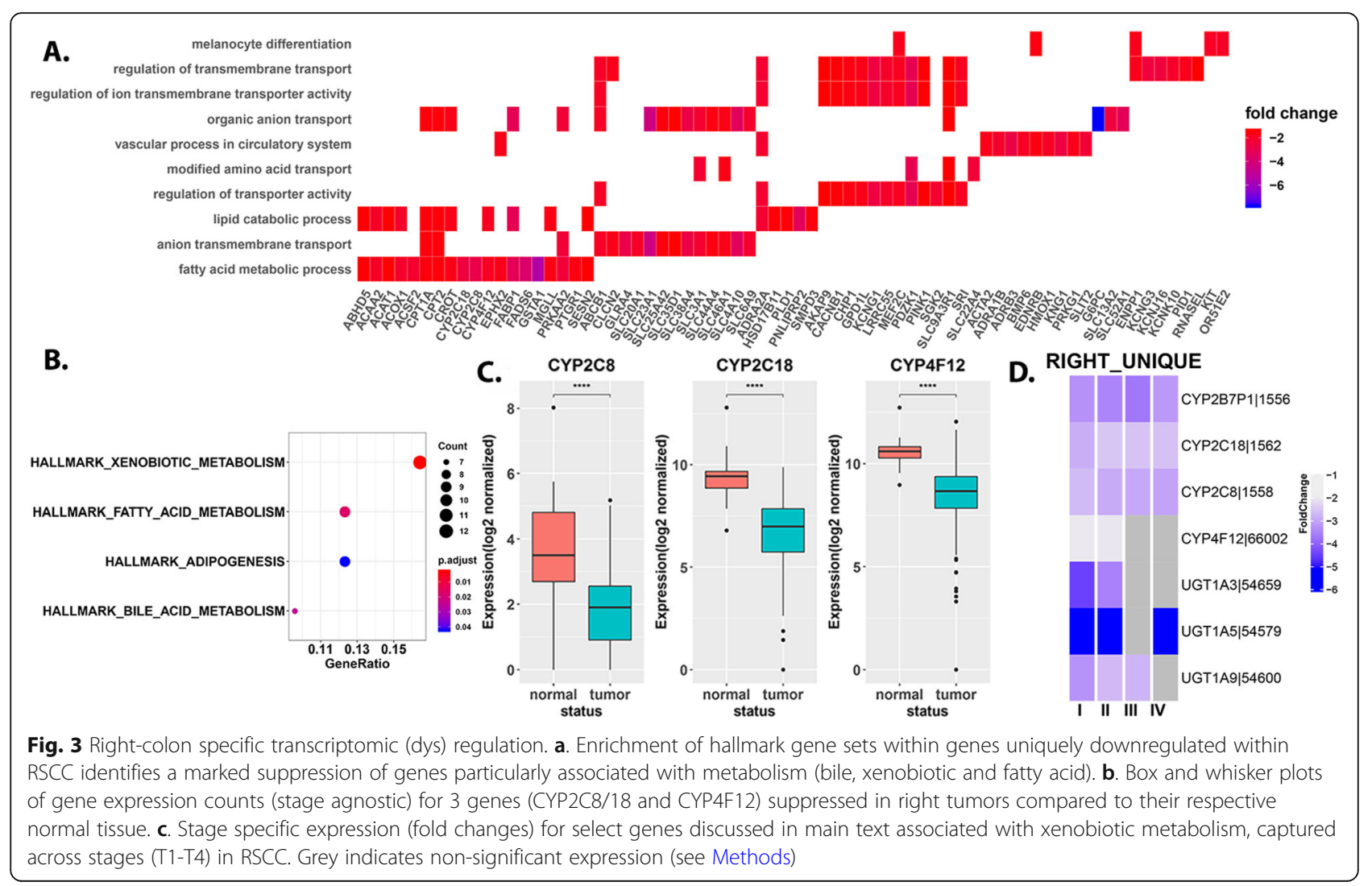

Proximal tumors predominantly exhibit dysregulation of UGT1A hepatic isoforms UGT1A3 and UGT1A9. Several solute carrier transporters particularly associated with drug (SLC25A42, SLC44A4, SLC46A1) and ascorbic acid transport $(S L C 23 A 1 / A 3)$ are also suppressed in RSCC. Stage specific analysis revealed a unique dysregulation of members from the CYP2C and UGT1A family within proximal tumors, specifically at early stages (T1-T2) (Fig. 3c).

Cancer cells preferentially use aerobic glycolysis to metabolize glucose, over mitochondrial oxidative phosphorylation (OXPHOS) characterized by increased glycolysis and lactate production. Our results suggest a more pronounced shift in metabolism in proximal tumors over distal tumors. The selective upregulation of SLC2A1 (GLUT1), a pivotal rate-limiting element in the transport and uptake of glucose combined with the unique downregulation of several mitochondrial metabolic markers involved in fatty acid degradation and oxidative phosphorylation including G6PC, FABP1, CPT1A, CPT2, ACAT1, ACAA2, ACOX1, EPHX2 and $E H H A D H$ further support the more pronounced shift in metabolism away from OXPHOS, within primary proximal tumors, asserting its more aggressive state [34].

\section{HOXB13 and SLC6A4 show opposing regulation trends in RSCC and LSCC}

Two genes appear to exhibit opposing regulation trends within RSCC and LSCC - HOXB13 and SLC6A4 (Fig. 4).
Nearly 95\% of the body's neurotransmitter-serotonin (5-hydroxytryptamine; 5-HT) is generated by the enterochromaffin cells, catalyzed by tryptophan hydroxylase $(T P H 1 / 2)$ within the intestine. Global loss-offunction studies for TPH1 have indicated an almost complete loss of intestinal 5-HT synthesis, implying that the observed suppression of TPH1 in both RSCC and LSCC indicates a curbed extracellular production of 5-HT [35]. Similarly, suppression of 5-HT receptors (e.g. HTR3E, HTR4) and intracellular enzymes required for breakdown of 5-HT (e.g. $M A O A, M A O B$ ) indicates a decreased bioavailability of 5 - $\mathrm{HT}$ in both LSCC and RSCC. In light of this, it is reasonable to observe a suppression of SLC6A4 (a ligand gated serotonin-selective reuptake transporter (SERT)), required for transport of 5-HT, such as in the case of RSCC. Though no evidence in literature exists for the differential role of $S L C 6 A 4$ in proximal or distal tumors within humans, we speculate that the observed upregulation of SERT expression within distal tumors (indicative of its increased activity), suggests alternate roles for SLC6A4 and/or mechanisms controlling its expression within LSCC.

On the other hand, $H O X B 13$ is an acknowledged oncogene. Studies geared specifically towards specific tumor location, have identified suppression of HOXB13 within distal tumors [36] and upregulation 

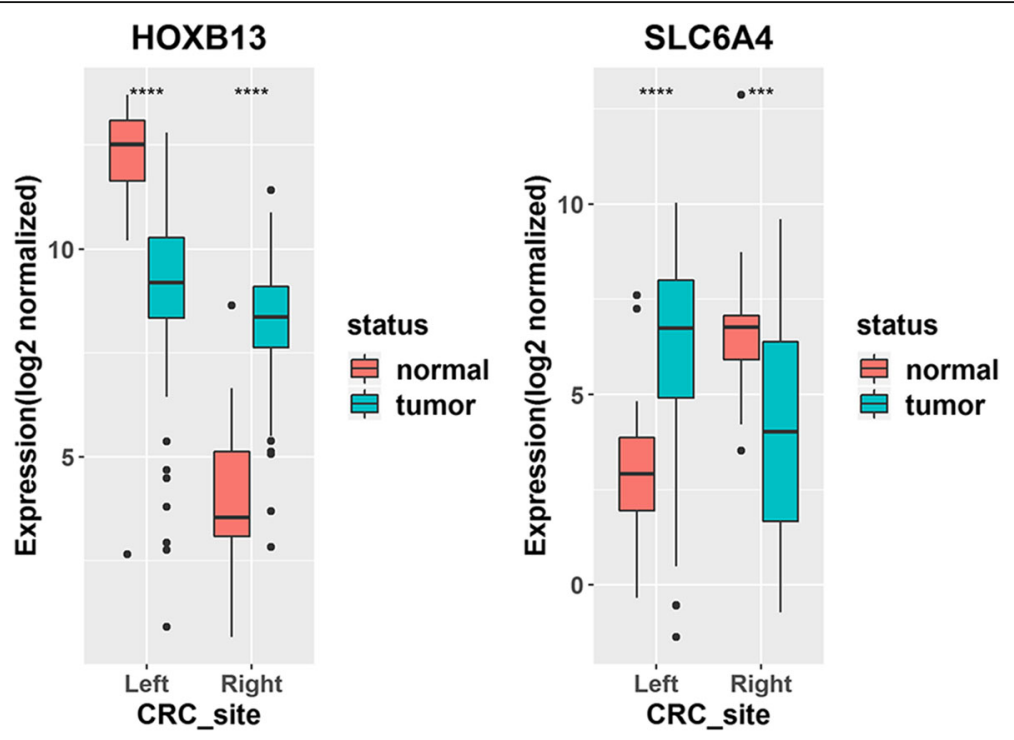

Fig. 4 Expression counts of HOXB13 and SLC6A4, within proximal (right) and distal (left) tumors and their corresponding normal tissue

within proximal tumors [37], consistent with our current analysis. Interestingly however, we also observe an upregulation of PRAC1 and PRAC2 (C17orf93), two genes genomically adjacent to HOXB13, within proximal tumors.

\section{Suppressed immune signaling predominates left-sided colon tumorigenesis}

Chemokines are expressed by various cell types, constitutively or under inflammatory conditions. Remarkably, distal tumors exhibit an enrichment of suppressed chemokine signaling, particularly B-cell and TFH markers, important immune infiltrates in colon cancer (Fig. 5). The role of B-cells, and its supporting cell types in immunosurveillance is complex and dichotomous. On one hand, animal models studies suggest participation in proliferation and metastasis by promoting chronic inflammation, and suppressing antitumor responses [38], while on the other hand, promote long term survival leading to increased intratumor densities of tumor infiltrating immune cells suppressing tumorigenesis [39]. MS4A1 (CD20, tumor infiltrating B-cell marker), and $B A C H 2$ (a well-known transcriptional regulator of $\mathrm{B}$ and TFH cells), two genes previously implicated in contributing to immune landscape differences between RSCC and LSCC [39], are more predominantly suppressed within distal tumors. Particularly interesting is the predominant downregulation of two chemokine signaling axes, within distal tumors (compared to normal) including the homeostatic chemokines CXCL13/CXCR5 (TFH cell markers) and CCL19/CCL21- CCR7 (migration and activation of immune cell types). Several of these markers including MS4A1, CXCR5, and CXCL13 are also suppressed across all stages (Stages 1-4) with respect to normal, further emphasizing their role in sustaining tumor behavior within LSCC (Fig. 5b and c).

In order to better understand the observed suppression of chemokine markers in a larger framework of regulation within distal tumors, we extracted clusters from a distal-specific protein interaction network (see Methods, Fig. 5d). We detected a large cluster of chemokines co-expressed with several G-protein couple receptor signaling and cAMP signaling proteins, including GRM8 (a cell surface marker in CRC), GNG2/4/7, $E D N 2 / 3$ and $A D C Y 2 / 5 / 9$ (adenylate cyclase). Two downregulated receptors LPAR1 (Lysophoatidic acid receptor), and CASR $\left(\mathrm{Ca}^{2+}\right.$ sensing receptor) involved in $\mathrm{Ca}^{2+}$ homeostasis, were also detected within this cluster [40, 41]. Taken together, these results lead us to speculate on a nexus between altered $\mathrm{Ca}^{2+}$ signaling mediated by GPCRs, specifically chemokines, and their subsequent impact on the inflammatory signatures within distal tumors (LSCC). Notably, 8/78 genes within this cluster including LPAR1, GNG4, GNG7, PMCH, GPR18, EDNRA, GPER1 and EDN3, (hypergeometric $p<0.07$ ), are sufficient to distinguish distal tumors, from normal distal colon as identified via recursive SVM classifier (see Methods).

RSCC exhibits pronounced post-transcriptional regulation Small non-translatable RNAs called miRNAs and several other RNA-binding proteins (RBPs), form an important class of molecules involved in post-transcriptional regulation (PTR). We focused on utilizing two levels of -omics data for analyzing differences in PTR within LSCC and RSCC. 


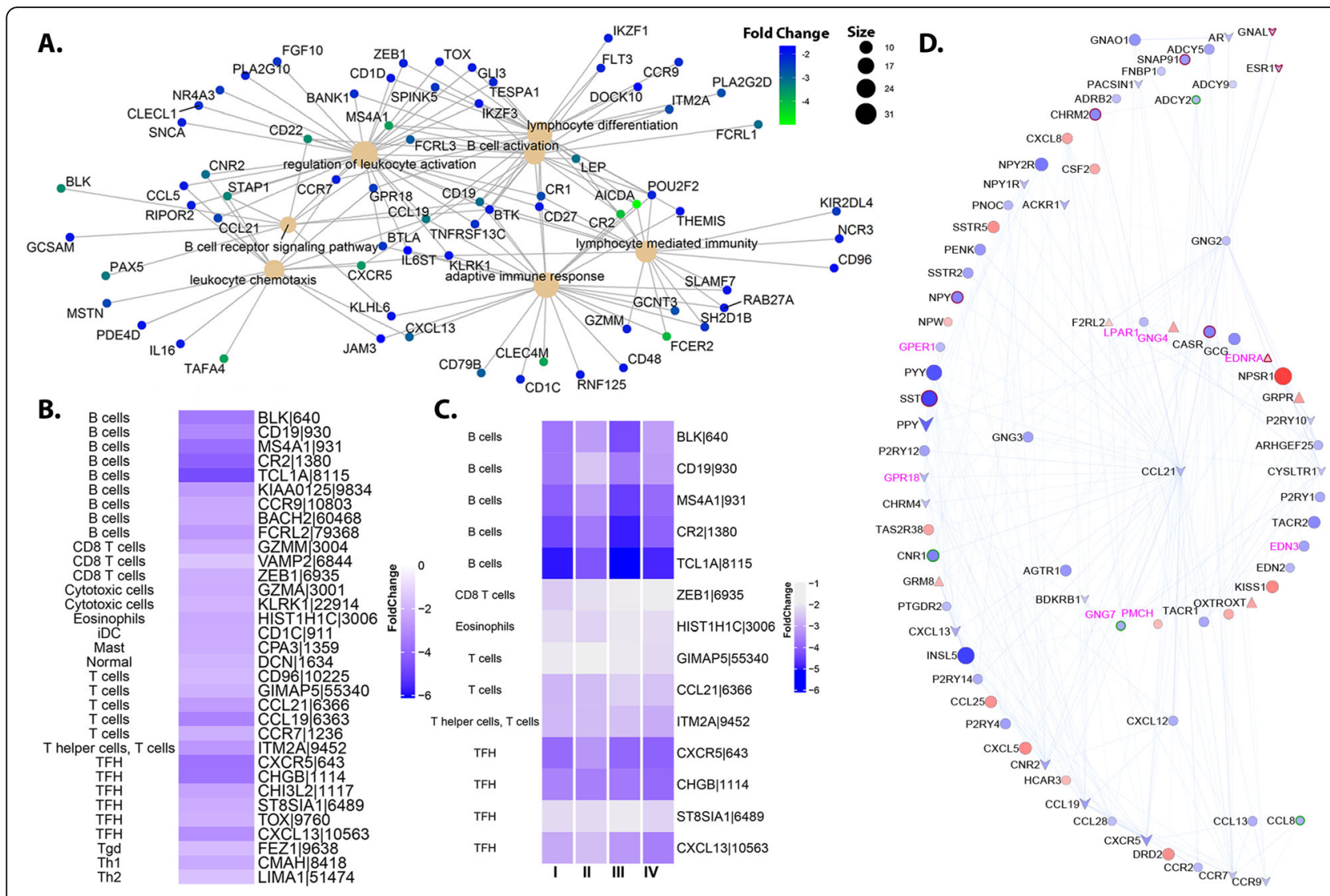

Fig. 5 Left-colon specific transcriptomic (dys)regulation. a. A graphical representation of suppressed gene functions (based on Gene Ontology) and its associated genes. Color on the gene nodes indicate their fold changes (compared to Left normal tissue) and size of functional nodes indicate the number of genes associated with that function. b. Fold changes of immune markers that are dysregulated within LSCC (stage agnostic) c. Stage specific capture of fold changes for immune markers that were suppressed across all stages of LSCC. $\mathbf{d}$. Protein interaction cluster (see Methods) which highlights the correlation patterns among GPCR and chemokines within LSCC. The color intensity is indicative of the strength of fold change going from light to dark (low to high fc) in either direction. Blue- downregulated, red-upregulated, triangle- uniquely upregulated, down arrow- uniquely downregulated, green border- hypomethylated, red border-hypermethylated

\section{Side-specific control of tumorigenesis by miRNAs}

Side-specific differential analysis of 1046 micro-RNAs (miRs) identified 325 differentially regulated miRs in RSCC and $200 \mathrm{miRs}$ in LSCC, compared to their respective normal tissue (see Methods, Supplementary table S8). A large majority of dysregulated miRs (198) are changing in both RSCC and LSCC. Several of the top commonly upregulated miRs are oncogenic-miRs including miR-135b, miR-577, miR-19a, miR-592 with roles in tumor initiation, proliferation/ progression and migration [42, 43]. Likewise, several miRs suppressed within both tumor types, including miR-328 [44], miR486 [45], have been previously indicated in inhibition of tumor progression in CRC.

Increasing evidence however suggests malleable roles for miRs, with multiple targets, amplifying their inhibitory or stimulatory effects on gene regulation through positive or negative feedback loops in conjunction with other miRs. We established functionally relevant, sidespecific miRNA-mRNA clusters (see Methods) in an effort to identify the influence of the differentially regulated miRs on gene expression. Analysis of clusters within RSCC revealed miRs regulating genes in interconnected pathways of cellular metabolism, cell growth and proliferation (Fig. 6a). For instance, uniquely upregulated miR-23a correlates with several mitochondrial proteins including G6PC and PPARGC1. Several miRs, particularly, miR-181d and miR-576, correlate with cell cycle genes including $B C L 2$ and $C C N D 1$. BCL2, a major regulator of mitochondrial apoptosis, has been consistently shown to be down regulated in colon (and cancer) [46]. Control of BCL2 expression via miR-24-2 (strongly upregulated in both proximal and distal tumors), has been previously reported in human embryonic kidney and breast cancer cell lines [47]. Interestingly, several uniquely regulated miRs correlate significantly with (hypermethylated) TWIST1, a primal transcription factor uniquely upregulated within proximal tumors [48], whose activation has been implicated in reverting cells to a non-lineage specific proliferative state. 


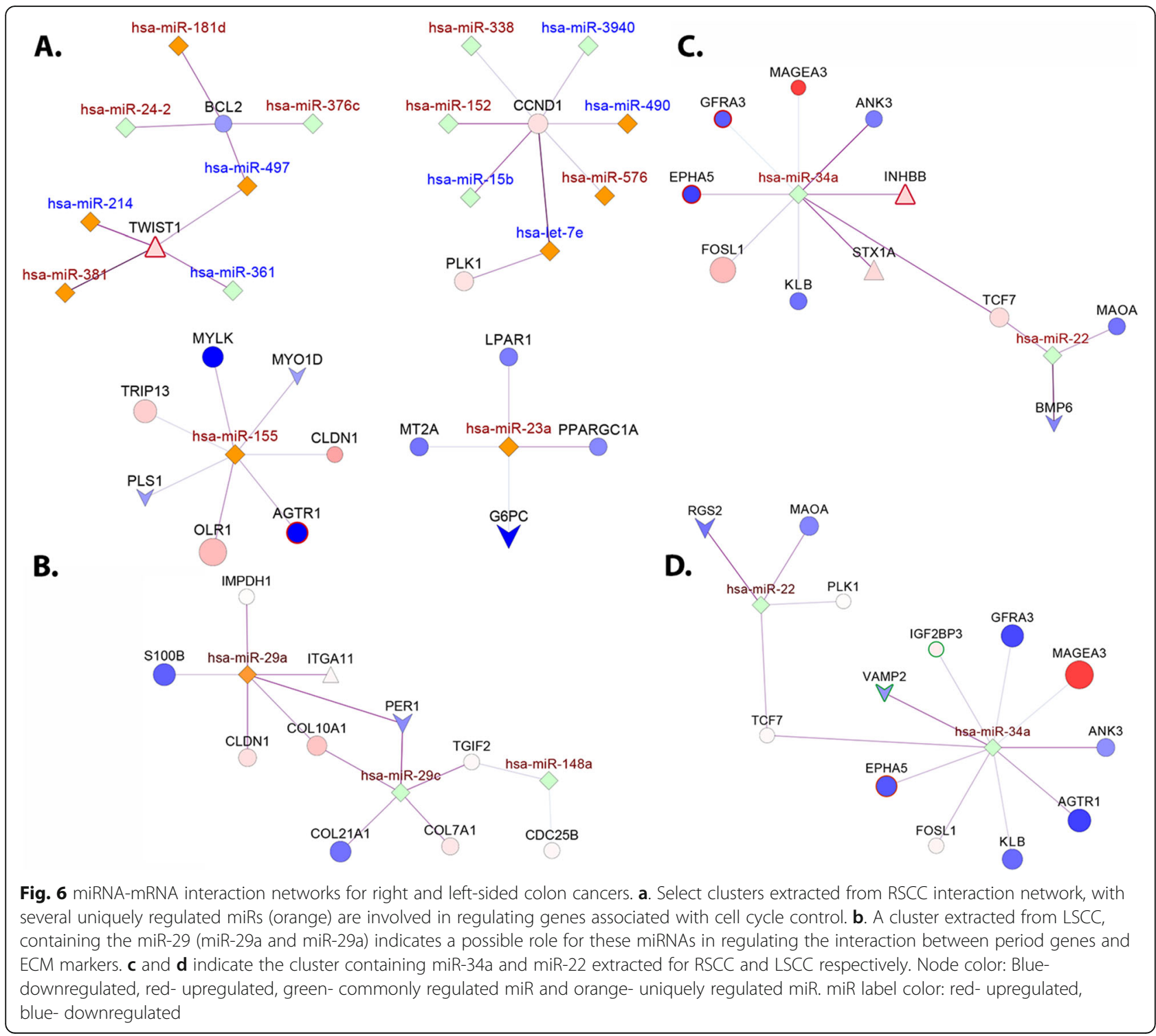

Only two miRs however, are uniquely regulated within distal tumors- miR-3607 and miR-29a (Fig. 6b). Interestingly, members of miR-29 family of oncomiRs (miR-29c and miR-29a) appeared to correlate with ECM and clock genes within distal tumors including downregulated PER1 (negative regulator of circadian rhythm) [49].

Particularly interesting are clusters conserved within both RSCC and LSCC (Fig. 6c and d). For example, miR-22 and miR-34a, two commonly regulated miRs in CRC, appear to cluster together. These miRs are known to impinge on processes of metabolism, angiogenesis, proliferation, migration, invasion, apoptosis and epithelial-to-mesenchymal transition (EMT) (a primary transformation for metastatic and invasive tumor cells. miR-34a (a tumor suppressor induced by p53 involved in EMT in CRC) $[50,51]$ correlates with several commonly regulated genes involved in signal transduction and EMT via the WNT and AKT signaling pathways including MAGEA3, GFRA3, EPHA5, $A N K 3$ and TCF7. The uniquely upregulated $I N H B B$, which correlates with miR-34a expression in proximal tumors (Fig. 6c), was also identified to be significantly associated with OS in RSCC (HR 95\% CI - 0.34 (0.18-0.65), logrank $p<0.001)$.

Differences in alternative splicing events mediated by RNAbinding proteins in LSCC and RSCC

Alternative splicing (AS) is an active PTR mechanism during which mRNA is actively rearranged accounting for the observed protein repertoire of complex organisms [17]. Utilizing Percent Splice-In (PSI) values from TCGASpliceSeq (see Methods), we identify 115 sigAS events among DEGs in RSCC and 101 sigAS events among DEGs in LSCC (see Methods). Exon skipping 
(ES), usage of alternate promoters (AP) and terminators (AT) were detected to be predominant and potent mechanisms for AS contributing to the etiology of colon cancers (Fig. 7a, Supplementary Table S10). Notably, our results indicate that a large proportion of the sigAS events $(n=64)$ occur in genes commonly dysregulated in both LSCC and RSCC, making alternative splicing a major PTR regulatory mechanism within colon cancers, including genes such as AXIN2 (ES, exon 7), and MXI1 (AP, exon 3) associated with the WNT pathway, and others such as IGF2 (AP, exon5), CXCL12 (AT, exon 5.2), CCL24 (AP, exon 1), and S100A2 (AP exon 3). SULT1A2 (RI, 1.2:1.3) and CALD1 (ES, 8.3:9) exhibit the highest $\triangle$ median PSI values between healthy and tumor tissues, in both left and right. SULT1A2 (suppressed $\sim 3$ $\log 2 \mathrm{fc}$ in both right and left tumors) is a sulfotransferase liver enzyme involved in detoxification of a variety of endogenous and xenobiotic compounds [52], while CALD1 is a novel target of TEA domain family member 4 involved in cell proliferation and migration (Fig. 7b and c). Missplicing of both these transcripts have been previously detected as events correlated with the etiology of disease [53, 54]. Particularly interesting are sigAS events that occur in a side specific manner, within genes uniquely regulated in either RSCC or LSCC, for instance, CYP4F12 ( $\Delta$ median PSI $=-0.19)$, UGT1A1 $(\Delta$ median $\mathrm{PSI}=0.12) S R I(\Delta$ median PSI $=-0.64)$ all exhibit significant AS within right tumors.
We identify a total of 76 and 66 RBPs to be differentially regulated in RSCC and LSCC, respectively. A large proportion (47 RBPs) are commonly regulated in both RSCC and LSCC with several enriched for binding among DEGs (adj $p<0.05$, see Methods), including RBPs previously discussed in the context of CRC such MSI2, MEX3A, IGF2BP1/3, ELVAL4 [55, 56], and cancers in general, such as RBM47, DKC1, CELF4, ELAVL3 (Fig. 7e, Supplementary Table S11). Downregulation of $R B M 47$ is involved in increased cell migration and invasion, and is indicated to promote EMT and metastasis within CRCs [57]. Notably, RBM47 is also significantly differentially spliced within both distal and proximal tumors, compared to normal tissues (AP, exon 2). However, a significant anti-correlation between its expression and PSI values is observed only within distal tumors (Fig. 7d), implying a possibility of feedback mechanisms controlling $R B M 47$ within distal tumors.

Additionally, we identify significant correlation between the expression of CELF4, RBM20, NOVA1 and PPARGC1A splicing associated RBPs and sigAS events in both proximal and distal tumors (see Methods). AFF2 is however uniquely associated within distal tumors. The resulting correlation network indicated that greater than $50 \%$ of sigAS events (66/101-left and 58/115) are correlated with these specific RBPs (adj $p<0.05$, Supplementary Figure 3), highlighting a possibly crucial role for them in the observed (many-to-one) regulation of transcripts within colon cancer.

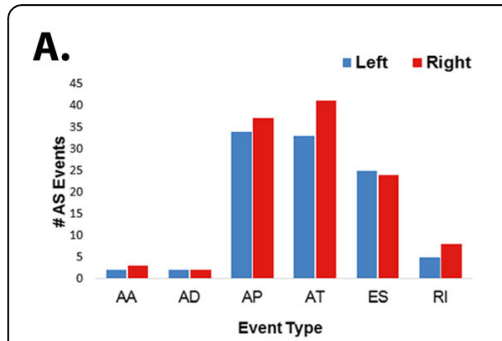

c.
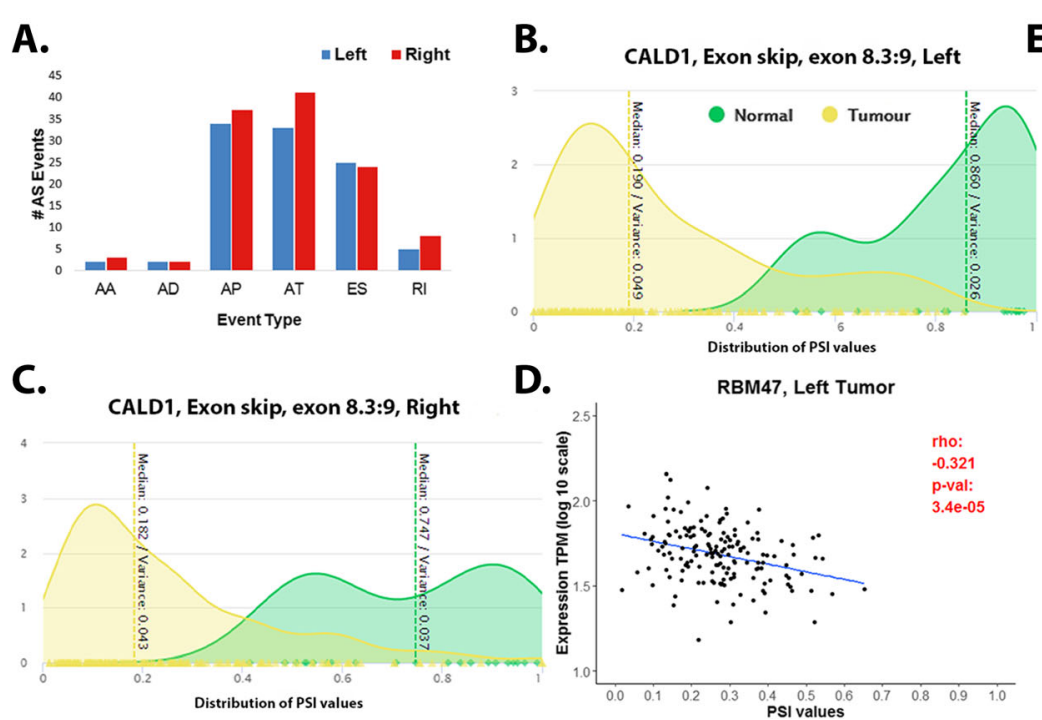

D.

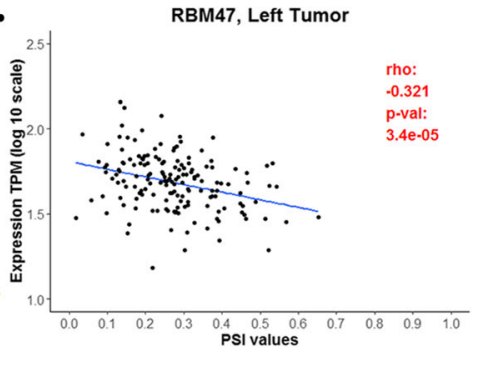

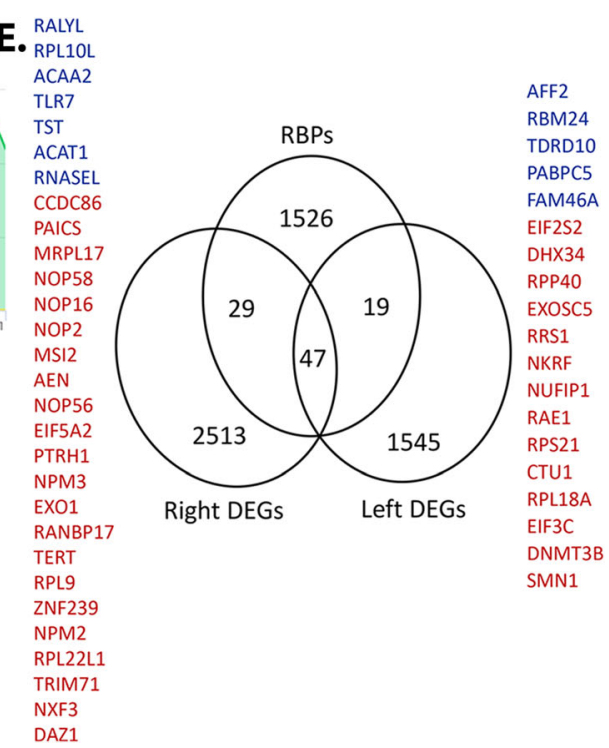

Fig. 7 Summary of post-transcriptional regulation. a. Number of AS events identified in left and right among DEGs (sigAS) events belonging each of the six event types (AA-Alternate acceptor, AD- alternate donor, AP- Alternate promoter, AT- Alternate terminator, ES- Exon skip, RI- Retained Intron). $\mathbf{b}$ and $\mathbf{c}$. Density plots for PSI value distributions of CALD1 gene in tumor and normal tissues for left and right respectively. D. Significant anti-correlation observed between expression of RBM47 (an RBP known to be suppressed in CRC) and the corresponding percent spliced-in (PSI) values within distal tumors. E. Venn highlighting RBPs identified among DEGs in LSCC and RSCC. RBPs identified as uniquely regulated on right (29 RBPs) and left (19 RBPs) are identified in color (blue-downregulated, red- upregulated) 
Differences in marker methylation and its association with gene expression, in RSCC and LSCC

Development and progression of colorectal cancer is understood to undergo several genetic and epigenetic changes. Changes in the DNA methylation is one of major epigenetic mechanisms controlling CRC [9]. Differential methylation analysis identified a larger proportion of hypermethylated CpG sites (DMPs) in proximal/ RSCC samples; while distal/LSCC exhibited a larger proportion of hypomethylated sites, compared to their controls respectively (see Methods, Fig. 8a). It is interesting to observe the genomic distribution indicated highest number of hypermethylated DMPs within the CpG Islands, while hypomethylation occurs in Open Seas (Fig. 8b). Previously published methylation markers including SEPT9, VIM, GATA4, INA, MAL, WNT (WNT2/ 2B/3/6/5A/7A, APC2) and CNRIP1 are hypermethylated in both RSCC and LSCC $[58,59]$, further establishing them as side-agnostic methylation markers of CRC.

We were additionally interested in identifying impact of differential methylation on DEGs and to this extent, extracted significant probe-gene pairs (both anticorrelated and correlated) from both RSCC and LSCC. We find that $33 \%$ of the downregulated genes are significantly anti-correlated with at least one hypermethylated probe and $27 \%$ with hypomethylated probes within distal tumors. On the other hand, we found a higher fraction of genes being controlled by differential methylation in proximal tumors $(\sim 40 \%$ of the downregulated/hypermethylated genes, and $\sim 20 \%$ upregulated/hypomethylated genes) indicative of a role for increased hypermethylation in suppressing expression with RSCC, consistent with prior research. Interestingly however, the hypermethylation and expression states of several

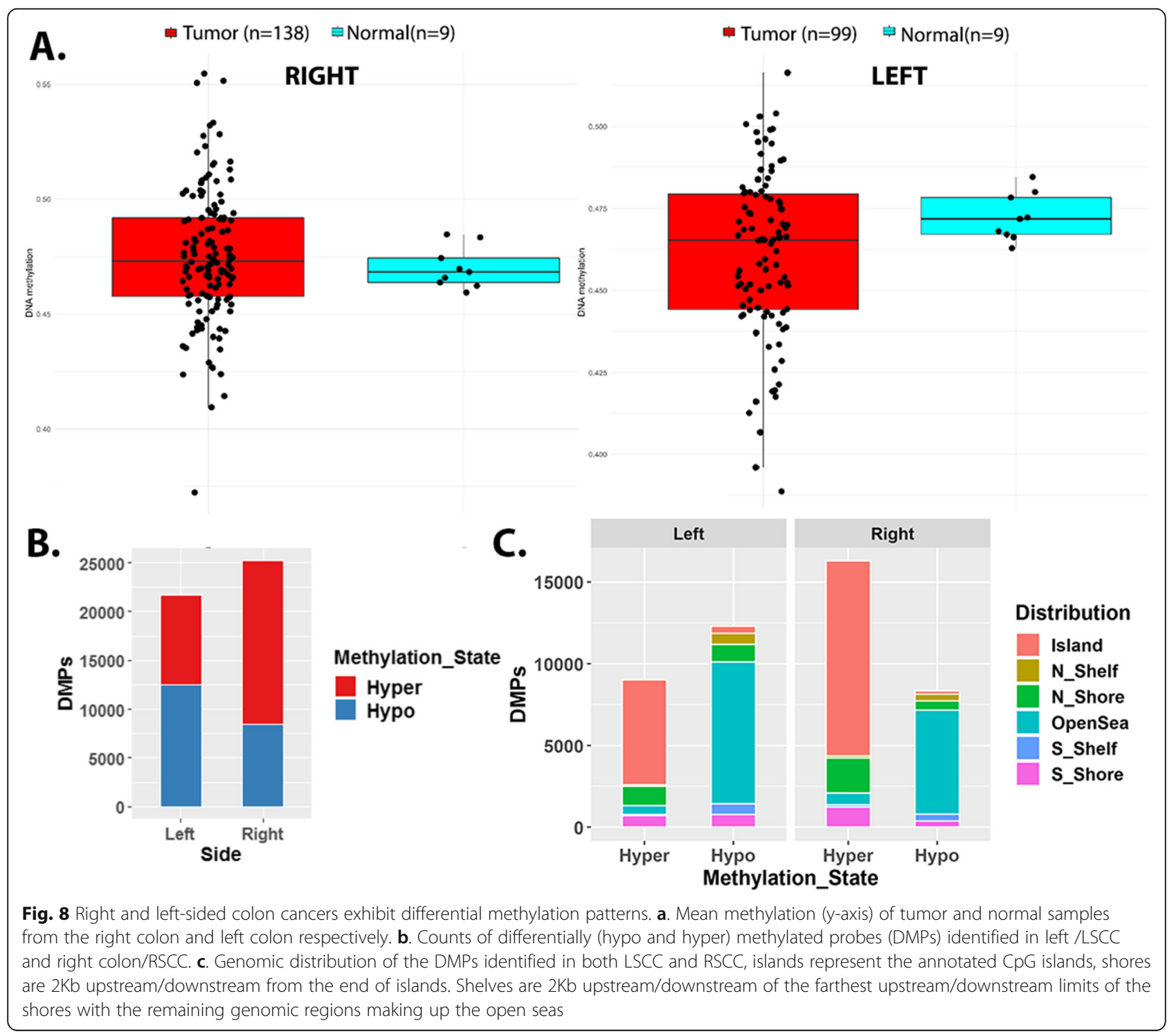


commonly regulated DEGs (such as OTOP2/3, CA1/2/4, NOTUM) is more obvious in LSCC than RSCC (Supplementary Table S9). Notably, we identified a significant enrichment of gene-probe pairs that exhibited positive correlation (overexpression and hypermethylation in tumors). For instance, $W N T 5 A / 2 / 3 / 7 B$ all exhibit significant correlation between expression and methylated DMPs in LSCC.

Changes in methylation state of region can be due to gain/loss of site-specific transcription factors [60]. We employed ELMER, to obtain insight into motifs and TFs which may be involved in setting tumor specific DNA methylation patterns within LSCC and RSCC (see Methods). In both these tumor types, we identify the FOSL1 binding motif to be highly ranked for hypomethylated/upregulated loci, indicating a possible gain of FOSL1 (significantly up $\sim 3 \mathrm{fc}$, in both), in a side independent manner. Likewise, downregulated/hypermethylated loci are enriched for the $S P 1 / 2 / 3$ binding motifs. TF factors including ISX (suppressed $\sim 2.3 \mathrm{fc}$ in both), contain these binding motifs, suggesting an observed loss of these site-specific TFs might dictate de-novo hypermethylation and suppression of its downstream targets, in a side independent manner within colon cancers.

\section{Discussion}

Proximal and distal colon tumors are suggested to be clinically, pathologically and transcriptionally distinct within CRC $[4,61]$. Multi-omics studies aimed at identifying differences between RSCC and LSCC have often targeted a direct comparison between the two tumor sides (e.g. [10]). However, we identify mechanistic differences in proximal and distal tumorigenesis by first comparing it to their respective normal tissues, within a conservatively chosen sample cohort. The results presented here illustrate the power of the study, to discriminate correlations in transcriptional and posttranscriptional regulation, which are likely lost in a direct comparison of the two tumor types, due to compensatory mechanisms in play. For instance, PRAC (PRAC1) is a heavily transcribed gene of the prostate, distal colon and rectum in normal physiology [62]. Studies in CRC, comparing RSCC with LSCC, have suggested a suppression of PRAC within proximal tumors [63]. On the contrary, our analysis indicates an overexpression of PRAC ( $4.6 \mathrm{fc}$ ), unique to RSCC, highlighting a possible functional role for PRAC within proximal tumors. Also, the observed upregulation of its adjacent genes (genomic co-ordinates), $H O X B 13$ and PRAC2 allude to possible co-regulatory mechanisms in RSCC [64], emphasizing their differential roles in proliferation and tumor growth potential within the two tumor types.
Pathways known to be altered in the pathogenesis of CRC, including WNT and MYC signaling, EMT transition, inflammatory responses (including TNF- $\alpha$ signaling via $N F k B$ ), and a suppression of KRAS signaling, were identified in both RSCC and LSCC. Rather than focusing on these traditionally well-known molecular mechanisms, we investigated mechanisms that contribute differentially to the sided-ness of colon cancer. Our findings indicate unique patterns of regulation, specifically within suppressed gene sets of RSCC and LSCC. A detailed analysis suggests a reduced capacity of RSCC to detoxify carcinogens, likely contributing to a genotoxic tumor environment, resulting in a more aggressive phenotype than LSCC. Additionally, increasing evidence of altered metabolism, a critical hallmark of colon cancer, is more evident in RSCC, further lending credence to the link between metabolism and cancer aggressiveness. A larger proportion of differentially regulated miRNAs on the right further support the observation that proximal tumors are more transcriptionally active [65]. Our results are particularly interesting in the context of LSCC, as they establish an enrichment of suppressed chemokine/GPCR signaling markers), at a transcriptional level. When explored within a larger context of dysregulation, using a network theoretic approach, our results highlight a crucial nexus between calcium homeostasis (sensing, mobilization and absorption, e.g. LPAR1 and CASR) and immune/GPCR signaling within LSCC patients, possibly contributing to the differential immune landscape of distal tumors, and their reduced proliferative and metastatic potential.

From a clinical standpoint, several tissue (invasive), and fecal and blood (non-invasive) markers are available for diagnosis and prognosis today [66, 67]. The power of our analysis lies in enabling identification of side and stage specific biomarkers. For instance, Cytokeratin (KRT7, KRT17 and KRT20) are often used as metastasis markers. Notably, we identify additional markers which exhibit preferential expression within distal and proximal tumors and may serve as putative markers for early vs late stage detection. For example, KRT31 (in early stages) and $K R T 40$ (in late stages) is expressed in distal tumors alone, while KRT13 and KRT15 are expressed in early stages of proximal tumors. We speculate KRT5, to be a stage-agnostic marker for RSCC. Likewise, FDA approved serum tumor marker CEA, is encoded by the CEACAM family of genes. Though, CEACAM1 and CEACAM7 are similarly upregulated in both LSCC and RSCC, CEACAM18- is predominantly upregulated in proximal tumors and may serve as a biomarker for RSCC.

PTR is a key regulatory step between transcription and translation [68]. RBPs and miRNAs are increasingly suggested to exhibit combinatorial regulation of themselves 
and their target mRNAs [69, 70] making delineating $\mathrm{RBP} /$ miRNA-target functional mapping a complex task. We highlight a handful of miRs including miR-29a (in LSCC); miR-155, miR181-d, miR-576 and miR23a (in RSCC), which may serve as side-specific markers of colon tumors, and as suitable candidates for future therapeutic research. Several highly regulated miRs including mir-3607, miR-3677, miR-3622a, miR-885, miR3620, miR-1295, miR-889, miR-653 with limited prior evidence for roles in colon cancer may serve as interesting targets for future studies, particularly in nonsynchronous tumors. We observe that a majority of AS events are mediated by select splicing associated RBPs within both tumors (LSCC and RSCC) including NOVA1, CELF4, and RBM20. Interestingly, NOVA1 knockdown was recently shown to significantly alter TERT transcripts in CRC [71]. Adding another layer to the complexity of PTR are opposing correlational trends between splicing factors and transcripts, suggesting target specific regulation by RBPs. For example, the increased splice-in of SULT2B1 (AP exon 1) observed in tumors, correlated negatively with the reduced expression of three splicing factors NOVA1, RBM20 and CELF4. Increased splice-in of CALD1 events within tumors (ES 8.3:9 and $A D, 8.3$ ) correlated significantly with the reduced expression of CELF4, NOVA1, and RBM2O in both left and right, suggesting differing impacts of differential regulation by these splicing factors.

Alterations in DNA methylation is an early event in cancer and has been suggested for use in non-invasive diagnosis within CRC $[58,59]$. Overall, our analysis of methylation data indicates that hypomethylation is a prominent phenomenon within distal tumors, in contrast to hypermethylation within proximal tumors. Analysis of differentially methylated loci established FOSL1 and $S P 1 / 2 / 3$ binding motifs as highly ranked for hypomethylated and hypermethylated genes respectively, in a side-independent manner within colon cancers. We postulate that ISX might serve as a major TF affecting hypermethylation dynamics within LSCC and RSCC. Additionally, our results indicate a positive correlation exists between epigenetic changes and gene expression of several genes, more prominently within LSCC. For instance, PHACTR3, a hypermethylated stool biomarker [72] in both tumors, shows a significant positive correlation between hypermethylation and (over) expression only within distal tumors. These instances further highlight a differential role for methylation and underscore alternate mechanisms of expression control within solid tumors, in a side-specific manner.

\section{Conclusion}

In this study, we analyze multi-omics data from colon cancer patients to decipher mechanisms of tumor etiology and progression, which contribute to the sidespecificity of colon cancers. We capitalize on publicly available data to identify distinctions in molecular signatures at an epigenetic (methylation), transcriptional (stage specific and stage-agnostic) and posttranscriptional levels (miRNA, alternative splicing mediated by RNA binding proteins) between right and left colon cancers. We show signatures associated with tumor aggressiveness arise from the genotoxic environment in RSCC and those with suppressed chemokine/ immune response are more prevalent in LSCC. Pronounced dysregulation of miRNAs and RBPs- two wellknown post-transcriptional regulators, on the right further strengthen the argument of a transcriptionally hyperactive and diverse RSCC. In addition to the differential methylation patterns, a surprising number of positive correlations (between differential methylation and expression) in both RSCC and LSCC underscore alternate mechanisms for expression control within colon tumors. Differences in functional mechanisms described here emphasize the molecular heterogeneity of colon cancers. Future experimental validation of the molecular players identified here, specifically within nonsynchronous tumors, will influence the efficacies of existing and future diagnostic (e.g. biomarker identification), prognostic (patient stratification and recurrence) and therapeutic (e.g. molecular) interventions.

\section{Supplementary information}

Supplementary information accompanies this paper at https://doi.org/10. 1186/s12885-020-06784-7.

\section{Additional file 1. An excel file containing all supplementary tables} highlighted with the manuscript as individual sheets.

Additional file 2: Figure 1. RSCC specific miR-mRNA interaction network clustered via MCL clustering. Validated interactions between differentially expressed genes and miRs were extracted from the miR-TAR database. The network was clustered on mRNA-miRNA co-expression strength using MCL clustering. Node color: Blue- downregulated, red- upregulated, greencommonly regulated $\mathrm{miR}$ and orange- uniquely regulated miR. miR label color: redupregulated, blue- downregulated. Figure $\mathbf{2}$ LSCC specific miR-mRNA interaction network clustered via MCL clustering. Validated interactions between differentially expressed genes and miRs were extracted from the miR-TAR database. The network was clustered on mRNA-miRNA co-expression strength using MCL clustering. Node color: Blue- downregulated, red- upregulated, green- commonly regulated $\mathrm{miR}$ and orange- uniquely regulated $\mathrm{miR}$. miR label color: redupregulated, bluedownregulated. Figure $\mathbf{3}$ - Correlation network of splicing factors and AS events- the correlation network for left and right tumors captures the correlation between splicing factors (differentially regulated $\mathrm{RBPS}$ ) and sigAS events occurring in each side. Blue edges indicated anti-correaltion, red-correlation. Pink nodes are the splicing factors, grey nodes indicate sigAS events. The shapes correspond to each of the six events identified and presented in legend within figure.

\section{Abbreviations}

5-HT: 5-hydroxytryptamine; AA: Arachidonic acid; AAs: Alternate acceptor sites; AD: Alternate donor sites; AP: Alternate promoter; AS: Alternative splicing; AT: Alternate terminator; CA: Carbonic anhydrases; CRC: Colorectal cancer; CIMP: CpG island methylation phenotype; DEGs: Differentially 
expressed genes; DEMs: Differentially expressed miRNAs; DMPs: Differentially methylated probes; EETs: Epoxyeicosatrienoic acids; EMT: Epithelial-tomesenchymal transition; ES: Exon skip; Gl: Gastrointestinal; LSCC: Left-sided colon cancers; miR: micro-RNAs; MSI: Microsatellite instability; ME: Mutually exclusive exons; NCCN: National Comprehensive Cancer Network; OS: Overall survival; OXOPHOS: mitochondrial oxidative phosphorylation; PPI: Proteinprotein interactions; PTR: Post-transcriptional regulation; RBP: RNA binding protein; RI: Retained intron; RSCC: Right-sided colon cancers;

SEER: Surveillance, Epidemiology and End Result; SERT: Serotonin-selective reuptake transporter; TCGA: The Cancer Genome Atlas; TF: Transcription factor; UGT: UDP-glucuronosyltransferase

\section{Acknowledgements}

Not Applicable.

\section{Authors' contributions}

Conception and design- KM, SS, SR; Data analysis - KM, NS; KM wrote the manuscript, and all authors have read and approved the final manuscript.

\section{Authors' information}

Not Applicable

\section{Funding}

National Institutes of Health, Grants: R01 DK109365, R01 HD084633, R01 HL106579, R01 HL108735, R01 LM012595, U01 CA198941, U01 CA200147, U01 DK097430, U19 Al090023, U2C DK119886; National Science Foundation Grant STC-0939370. The funding bodies were not directly involved in analyses, and interpretation of data or in the writing of the manuscript.

\section{Availability of data and materials}

The datasets analyzed in the current study are all publicly available in the Genomic data commons (GDC) repository, [https://portal.gdc.cancer.gov/ projects/TCGA-COAD]. No explicit permission was required to download level 3 data for TCGA-COAD from Firebrowse.

\section{Ethics approval and consent to participate}

Not Applicable.

\section{Consent for publication}

Not Applicable.

\section{Competing interests}

The authors declare that they have no competing interests.

\section{Author details}

${ }^{1}$ Department of Bioengineering, University of California, San Diego, La Jolla, CA, USA. ${ }^{2}$ Division of Colon and Rectal Surgery, Moores Cancer Center, University of California San Diego Health System, La Jolla, CA, USA. ${ }^{3}$ Department of Cellular and Molecular Medicine, University of California, San Diego, La Jolla, CA, USA. " Department of Computer Science and Engineering, University of California, San Diego, La Jolla, CA, USA.

\section{Received: 7 January 2020 Accepted: 24 March 2020}

\section{Published online: 15 April 2020}

\section{References}

1. Fearon ER. Molecular genetics of colorectal cancer. Annu Rev Pathol. 2011;6: 479-507.

2. Guinney J, Dienstmann $R$, Wang $X$, de Reyniès $A$, Schlicker A, Soneson C, et al. The consensus molecular subtypes of colorectal Cancer. Nat Med. 2015;21:1350-6.

3. The Cancer Genome Atlas Network. Comprehensive molecular characterization of human colon and rectal cancer. Nature. 2012;487:330-7.

4. Mik M, Berut M, Dziki L, Trzcinski R, Dziki A. Right- and left-sided colon cancer - clinical and pathological differences of the disease entity in one organ. Arch Med Sci AMS. 2017;13:157-62.

5. NCCN guidelines for treatment of cancer by site. NCCN guidelines for treatment of cancer by site. https://www.nccn.org/professionals/physician_ gls/pdf/colon.pdf. Accessed 11 Oct 2019.
6. Narayanan S, Gabriel E, Attwood K, Boland P, Nurkin S. Association of Clinicopathologic and Molecular Markers on stage-specific survival of right versus left Colon Cancer. Clin Colorectal Cancer. 2018;17:e671-8.

7. Yahagi M, Okabayashi K, Hasegawa H, Tsuruta M, Kitagawa Y. The worse prognosis of right-sided compared with left-sided Colon cancers: a systematic review and meta-analysis. J Gastrointest Surg Off J Soc Surg Aliment Tract. 2016;20:648-55.

8. Zhang L, Zhao Y, Dai Y, Cheng J-N, Gong Z, Feng Y, et al. Immune landscape of colorectal Cancer tumor microenvironment from different primary tumor location. Front Immunol. 2018;9. https://doi.org/10.3389/ fimmu.2018.01578.

9. Koestler DC, Li J, Baron JA, Tsongalis GJ, Butterly LF, Goodrich M, et al. Distinct patterns of DNA methylation in conventional adenomas involving the right and left colon. Mod Pathol Off J U S Can Acad Pathol Inc. 2014;27. https://doi.org/10.1038/modpathol.2013.104.

10. Hu W, Yang Y, Li X, Huang M, Xu F, Ge W, et al. Multi-omics approach reveals distinct differences in left-and right-sided colon cancer. Mol Cancer Res. 2018;16:476-85.

11. lacopetta B. Are there two sides to colorectal cancer? Int J Cancer. 2002;101: 403-8.

12. Gentleman RC, Carey VJ, Bates DM, Bolstad B, Dettling M, Dudoit S, et al. Bioconductor: open software development for computational biology and bioinformatics. Genome Biol. 2004;5:R80.

13. Zhou X, Tuck DP. MSVM-RFE: extensions of SVM-RFE for multiclass gene selection on DNA microarray data. Bioinformatics. 2007;23:1106-14.

14. Colaprico A, Silva TC, Olsen C, Garofano L, Cava C, Garolini D, et al. TCGAbiolinks: an R/bioconductor package for integrative analysis of TCGA data. Nucleic Acids Res. 2016;44:e71.

15. Chou C-H, Shrestha S, Yang C-D, Chang N-W, Lin Y-L, Liao K-W, et al. miRTarBase update 2018: a resource for experimentally validated microRNAtarget interactions. Nucleic Acids Res. 2017;46:D296-302.

16. Saraiva-Agostinho N, Barbosa-Morais NL. Psichomics: graphical application for alternative splicing quantification and analysis. Nucleic Acids Res. 2019; 47:e7.

17. Wang Y, Liu J, Huang B, Xu Y, Li J, Huang L, et al. Mechanism of alternative splicing and its regulation. Biomed Rep. 2015;3:152-8.

18. Hentze MW, Castello A, Schwarzl T, Preiss T. A brave new world of RNAbinding proteins. Nat Rev Mol Cell Biol. 2018;19:327.

19. Gerstberger S, Hafner M, Tuschl T. A census of human RNA-binding proteins. Nat Rev Genet. 2014;15:829-45.

20. Dassi E, Re A, Leo S, Tebaldi T, Pasini L, Peroni D, et al. AURA 2: empowering discovery of post-transcriptional networks. Translation. 2014;2:e27738.

21. Silva TC, Coetzee SG, Gull N, Yao L, Hazelett DJ, Noushmehr H, et al. ELMER v.2: an R/bioconductor package to reconstruct gene regulatory networks from DNA methylation and transcriptome profiles. Bioinforma Oxf Engl. 2019;35:1974-7.

22. Shannon P, Markiel A, Ozier O, Baliga NS, Wang JT, Ramage D, et al. Cytoscape: a software environment for integrated models of biomolecular interaction networks. Genome Res. 2003;13:2498.

23. Yu G, Wang L-G, Han Y, He Q-Y. clusterProfiler: an R package for comparing biological themes among gene clusters. OMICS J Integr Biol. 2012;16:284-7.

24. Kuleshov MV, Jones MR, Rouillard AD, Fernandez NF, Duan Q, Wang Z, et al. Enrichr: a comprehensive gene set enrichment analysis web server 2016 update. Nucleic Acids Res. 2016;44 Web Server issue:W90-7.

25. De Robertis M, Arigoni M, Loiacono L, Riccardo F, Calogero RA, Feodorova $Y$, et al. Novel insights into Notum and glypicans regulation in colorectal cancer. Oncotarget. 2015;6:41237.

26. Yoon JH, Kim D, Kim J, Lee H, Ghim J, Kang BJ, et al. NOTUM is involved in the progression of colorectal Cancer. Cancer Genomics Proteomics. 2018;15: 485-97.

27. Weon $\mathrm{J}$, Potts PR. The MAGE protein family and cancer. Curr Opin Cell Biol. 2015;37:1-8.

28. Mboge MY, Mahon BP, McKenna R, Frost SC. Carbonic anhydrases: role in pH control and Cancer. Metabolites. 2018;8. https://doi.org/10.3390/ metabo8010019.

29. Okuno K, Matsubara T, Nakamura T, lino T, Kakimoto T, Asanuma K, et al. Carbonic anhydrase IX enhances tumor cell proliferation and tumor progression in osteosarcoma. OncoTargets Ther. 2018;11:6879-86.

30. Qu H, Su Y, Yu L, Zhao H, Xin C. Wild-type p53 regulates OTOP 2 transcription through DNA loop alteration of the promoter in colorectal cancer. FEBS Open Bio. 2019;9(1):26-34. 
31. Beyerle J, Frei E, Stiborova M, Habermann N, Ulrich CM. Biotransformation of xenobiotics in the human colon and rectum and its association with colorectal cancer. Drug Metab Rev. 2015;47:199-221.

32. Wang W, Yang J, Edin ML, Wang Y, Luo Y, Wan D, et al. Targeted metabolomics identifies the cytochrome P450 Monooxygenase eicosanoid pathway as a novel therapeutic target of Colon tumorigenesis. Cancer Res. 2019;79:1822-30.

33. Wang M, Sun D-F, Wang S, Qing Y, Chen S, Wu D, et al. Polymorphic expression of UDP-glucuronosyltransferase UGTIA gene in human colorectal cancer. PLoS One. 2013;8:e57045.

34. DeBerardinis RJ, Chandel NS. Fundamentals of cancer metabolism. Sci Adv. 2016;2:e1600200.

35. Terry N, Margolis KG. Serotonergic mechanisms regulating the Gl tract: experimental evidence and therapeutic relevance. Handb Exp Pharmacol. 2017;239:319-42.

36. Jung C, Kim R-S, Zhang H, Lee S-J, Sheng H, Loehrer PJ, et al. HOXB13 is downregulated in colorectal cancer to confer TCF4-mediated transactivation. Br J Cancer. 2005;92:2233-9.

37. Tatangelo F, Di Mauro A, Scognamiglio G, Aquino G, Lettiero A, Delrio P, et al. Posterior HOX genes and HOTAIR expression in the proximal and distal colon cancer pathogenesis. J Transl Med. 2018;16:350.

38. Zhu Z, Zhang X, Guo H, Fu L, Pan G, Sun Y. CXCL13-CXCR5 axis promotes the growth and invasion of colon cancer cells via PI3K AKT pathway. Mol Cell Biochem. 2015;400:287-95.

39. Bindea G, Mlecnik B, Tosolini M, Kirilovsky A, Waldner M, Obenauf AC, et al. Spatiotemporal dynamics of intratumoral immune cells reveal the immune landscape in human cancer. Immunity. 2013;39:782-95.

40. lamartino L, Elajnaf T, Kallay E, Schepelmann M. Calcium-sensing receptor in colorectal inflammation and cancer: current insights and future perspectives. World J Gastroenterol. 2018:24:4119-31.

41. Yang M, Zhong WW, Srivastava N, Slavin A, Yang J, Hoey T, et al. G proteincoupled lysophosphatidic acid receptors stimulate proliferation of colon cancer cells through the $\beta$-catenin pathway. Proc Natl Acad Sci U S A. 2005;102:6027-32.

42. Masuda T, Hayashi N, Kuroda Y, Ito S, Eguchi H, Mimori K. MicroRNAs as biomarkers in colorectal Cancer. Cancers. 2017;9. https://doi.org/10.3390/cancers9090124.

43. Magalhães L, Quintana LG, Lopes DCF, Vidal AF, Pereira AL, D'Araujo Pinto LC, et al. APC gene is modulated by hsa-miR-135b-5p in both diffuse and intestinal gastric cancer subtypes. BMC Cancer. 2018;18:1055.

44. Xu XT, Xu Q, Tong JL, Zhu MM, Nie F, Chen X, et al. MicroRNA expression profiling identifies miR-328 regulates cancer stem cell-like SP cells in colorectal cancer. Br J Cancer. 2012:106:1320-30.

45. Zhang Y, Fu J, Zhang Z, Qin H. miR-486-5p regulates the migration and invasion of colorectal cancer cells through targeting PIK3R1. Oncol Lett. 2018;15:7243-8.

46. Kaklamanis L, Savage A, Whitehouse R, Doussis-Anagnostopoulou I, Biddolph S, Tsiotos $\mathrm{P}$, et al. Bcl-2 protein expression: association with p53 and prognosis in colorectal cancer. Br J Cancer. 1998;77:1864-9.

47. Singh R, Saini N. Downregulation of BCL2 by miRNAs augments druginduced apoptosis - a combined computational and experimental approach. J Cell Sci. 2012;125:1568-78.

48. Nairismägi M-L, Füchtbauer A, Labouriau R, Bramsen JB, Füchtbauer E-M. The proto-oncogene TWIST1 is regulated by microRNAs. PLoS One. 2013;8:e66070.

49. Krugluger W, Brandstaetter A, Kállay E, Schueller J, Krexner E, Kriwanek S, et al. Regulation of genes of the circadian clock in human colon cancer: reduced period-1 and dihydropyrimidine dehydrogenase transcription correlates in high-grade tumors. Cancer Res. 2007;67:7917-22.

50. Slabáková E, Culig Z, Remšík J, Souček K. Alternative mechanisms of miR-34a regulation in cancer. Cell Death Dis. 2017;8:e3100.

51. Rokavec M, Öner MG, Li H, Jackstadt R, Jiang L, Lodygin D, et al. IL-6R/ STAT3/miR-34a feedback loop promotes EMT-mediated colorectal cancer invasion and metastasis. J Clin Invest. 2014;124:1853-67.

52. Glatt $\mathrm{H}$, Engelke $\mathrm{CE}$, Pabel U, Teubner W, Jones AL, Coughtrie MW, et al. Sulfotransferases: genetics and role in toxicology. Toxicol Lett. 2000;1 12:341-8.

53. Thorsen K, Sørensen KD, Brems-Eskildsen AS, Modin C, Gaustadnes M, Hein A-MK, et al. Alternative splicing in colon, bladder, and prostate cancer identified by exon array analysis. Mol Cell Proteomics. 2008;7:1214-24.

54. Saferali A, Yun JH, Parker MM, Sakornsakolpat P, Chase RP, Lamb A, et al. Analysis of genetically driven alternative splicing identifies FBXO38 as a novel COPD susceptibility gene. PLoS Genet. 2019;15:e1008229.

55. Chatterji P, Rustgi AK. RNA binding proteins in intestinal epithelial biology and colorectal cancer. Trends Mol Med. 2018:24:490-506.
56. García-Cárdenas JM, Guerrero S, López-Cortés A, Armendáriz-Castillo I, GuevaraRamírez P, Pérez-Villa A, et al. Post-transcriptional regulation of colorectal Cancer: a focus on RNA-binding proteins. Front Mol Biosci. 2019;6:65.

57. Rokavec M, Kaller M, Horst D, Hermeking H. Pan-cancer EMT-signature identifies RBM47 down-regulation during colorectal cancer progression. Sci Rep. 2017;7:1-15.

58. Lind GE, Danielsen SA, Ahlquist T, Merok MA, Andresen K, Skotheim Rl, et al. Identification of an epigenetic biomarker panel with high sensitivity and specificity for colorectal cancer and adenomas. Mol Cancer. 2011;10:85.

59. Hashimoto Y, Zumwalt TJ, Goel A. DNA methylation patterns as noninvasive biomarkers and targets of epigenetic therapies in colorectal cancer. Epigenomics. 2016;8:685-703.

60. Zhu H, Wang G, Qian J. Transcription factors as readers and effectors of DNA methylation. Nat Rev Genet. 2016;17:551-65.

61. Missiaglia E, Jacobs B, D'ario G, Di Narzo A, Soneson C, Budinska E, et al. Distal and proximal colon cancers differ in terms of molecular, pathological, and clinical features. Ann Oncol. 2014;25:1995-2001.

62. Liu XF, Olsson P, Wolfgang CD, Bera TK, Duray P, Lee B, et al. PRAC: a novel small nuclear protein that is specifically expressed in human prostate and colon. Prostate. 2001;47:125-31.

63. Bauer KM, Hummon AB, Buechler S. Right-side and left-side colon cancer follow different pathways to relapse. Mol Carcinog. 2012;51:411-21.

64. Xiong Y, Kuang W, Lu S, Guo H, Wu M, Ye M, et al. Long noncoding RNA HOXB13-AS1 regulates $\mathrm{HOXB13}$ gene methylation by interacting with $\mathrm{EZH} 2$ in glioma. Cancer Med. 2018;7:4718-28.

65. Ell B, Kang Y. Transcriptional control of cancer metastasis. Trends Cell Biol. 2013;23:603-11.

66. Nikolouzakis TK, Vassilopoulou L, Fragkiadaki P, Mariolis Sapsakos T, Papadakis GZ, Spandidos DA, et al. Improving diagnosis, prognosis and prediction by using biomarkers in CRC patients (review). Oncol Rep. 2018; 39:2455-72.

67. Lopez NE, Weiss AC, Robles J, Fanta P, Ramamoorthy SL. A systematic review of clinically available gene expression profiling assays for stage ॥ colorectal cancer: initial steps toward genetic staging. Am J Surg. 2016;212: 700-14.

68. Kornblihtt AR, Schor IE, Alló M, Dujardin G, Petrillo E, Muñoz MJ. Alternative splicing: a pivotal step between eukaryotic transcription and translation. Nat Rev Mol Cell Biol. 2013;14:153-65.

69. Dassi E. Handshakes and fights: the regulatory interplay of RNA-binding proteins. Front Mol Biosci. 2017;4:67.

70. O'Brien J, Hayder H, Zayed Y, Peng C. Overview of microRNA biogenesis, mechanisms of actions, and circulation. Front Endocrinol. 2018:9:402.

71. Ludlow AT, Wong MS, Robin JD, Batten K, Yuan L, Lai T-P, et al. NOVA1 regulates hTERT splicing and cell growth in non-small cell lung cancer. Nat Commun. 2018;9:1-15

72. Bosch LJW, Oort FA, Neerincx M, Khalid-de Bakker CAJ, Terhaar sive Droste JS, Melotte $V$, et al. DNA methylation of phosphatase and actin regulator 3 detects colorectal cancer in stool and complements FIT. Cancer Prev Res Phila Pa. 2012:5:464-72.

\section{Publisher's Note}

Springer Nature remains neutral with regard to jurisdictional claims in published maps and institutional affiliations.

Ready to submit your research? Choose BMC and benefit from:

- fast, convenient online submission

- thorough peer review by experienced researchers in your field

- rapid publication on acceptance

- support for research data, including large and complex data types

- gold Open Access which fosters wider collaboration and increased citations

- maximum visibility for your research: over $100 \mathrm{M}$ website views per year

At BMC, research is always in progress.

Learn more biomedcentral.com/submissions 